(NASA-TM-110074) MEASUREMENT OF

N95-16577

RESIDUAL FLEXIBILITY FOR

SUBSTRUCTURES HAVING PROMINENT

FLEXIBLE INTERFACES (NASA.

Marshall Space Flight Center) $18 \mathrm{p}$

Unclas

G3E:390033803 


\title{
ABSTRACT \\ MEASUREMENT OF RESIDUAL FLEXIBILITY FOR SUBSTRUCTURES HAVING PROMINENT FLEXIBLE INTERFACES
}

\author{
Michael L. Tinker and Paul S. Bookout \\ NASA/MSFC ED26 \\ Marshall Space Flight Center, AL 35812
}

(205) 544-4973

\section{Introduction}

Verification of a dynamic model of a constrained structure requires a modal survey test of the physical structure and subsequent modification of the model to obtain the best agreement possible with test data. Constrained-boundary or fixed-base testing has historically been the most common approach for verifying constrained mathematical models, since the boundary conditions of the test article are designed to match the actual constraints in service. However, there are difficulties involved with fixed-base testing, in some cases making the approach impractical. As stated in Refs. 1 and 2, it is not possible to conduct a truly fixed-base test due to coupling between the test article and the fixture. In addition, it is often difficult to accurately simulate the actual boundary constraints, and the cost of designing and constructing the fixture may be prohibitive. For use when fixed-base testing proves impractical or undesirable, alternate free-boundary test methods have been investigated, including the residual flexibility technique. The residual flexibility approach has been treated analytically in considerable detail (Refs. $3-5,7$ ) and has had limited application as a test method (Refs. 6-8).

Some investigators have expressed concern over difficulty in performing the required frequency response measurements for the method. This concern is well-justified for a number of reasons. Two of these are well-described by Blair in Ref. 9. First, residual flexibilities are very small numbers, typically on the order of $1.0 \mathrm{E}-6 \mathrm{in} / \mathrm{lb}$ for translational diagonal terms, and orders of magnitude smaller for off-diagonal values. This poses difficulty in obtaining accurate and noise-free measurements, especially for points removed from the excitation source. A second difficulty encountered in residual measurements lies in obtaining a clean residual function in the process of subtracting synthesized modal data from a measured response function. Inaccuracies occur since modes are not subtracted exactly, but only to the accuracy of the curve fits for each mode; these errors are compounded with increasing distance from the excitation point.

In this paper, the residual flexibility method is applied to a simple structure in both test and analysis. Measured and predicted residual functions are compared, and regions of poor data in the measured curves are described. It is found that for accurate residual measurements, frequency response functions having prominent stiffness lines in the acceleration/force format are needed. The lack of such stiffness lines increases measurement errors. Interface drive point frequency response functions for shuttle orbiter payloads exhibit dominant stiffness lines, making the residual test approach a good candidate for payload modal tests when constrained tests are inappropriate. Difficulties in extracting a residual flexibility value from noisy test data are discussed. It is shown that use of a weighted second order least-squares curve fit of the measured residual function allows identification of residual flexibility that compares very well with predictions for the simple structure. This approach also provides an estimate of second order residual mass effects. 


\section{Background of the Residual Flexibility Approach}

The technique of using an approximation of the effects of neglected higher order modes, or residual modes, to improve the accuracy of reduced-basis mathematical models was first presented by MacNeal (Ref. 3). In MacNeal's method, a substructure model derived from truncated modal properties was improved by including additional elements derived from first-order static approximations of the effects of higher modes. Rubin (Ref. 4) used a special statics problem to derive an expression for residual flexibility in a form that is more easily applied in structural dynamic analyses. As described in Ref. 4, the flexible-body displacements for a substructure can be written as a first-order approximation of residual effects,

$$
\mathbf{u}_{\mathrm{f}}=\mathbf{G F}=\mathbf{A}^{\mathrm{T}} \mathbf{G}_{\mathrm{c}} \mathbf{A F}
$$

where $\mathbf{G}$ is the free-free flexibility matrix. The constrained flexibility matrix is $\mathbf{G}_{c}$ and the transformation matrix $A=I-M \Phi_{R} M_{R}^{-1} \Phi_{R}^{T}$. It is noted that $M_{R}$ is the generalized mass associated with the rigid body modes $\Phi_{R}$. If the contribution of modes to be retained is removed from the deflection for the flexible substructure, the residual flexibility matrix $\mathbf{G}$ results, as shown in Eq. (2):

$$
\mathbf{u}_{\mathrm{fr}}=\left(\mathbf{G}-\mathbf{G}_{\mathrm{n}}\right) \mathbf{F}=\mathbf{G}_{\mathrm{r}} \mathbf{F}
$$

where $G_{n}=\Phi_{n} K_{n}^{-1} \Phi_{n}^{T}$ is the flexibility matrix corresponding to the retained modes.

In Ref. 5, Martinez, et. al, expressed substructure displacements in the form

$$
\mathbf{u}=\Phi \mathbf{q}+\mathbf{G}_{\mathbf{r b}_{\mathrm{b}}} \mathbf{F}_{\mathrm{b}}=\left[\begin{array}{ll}
\Phi & \mathbf{G}_{\mathrm{rb}_{\mathrm{b}}}
\end{array}\right]\left\{\begin{array}{c}
\mathbf{q} \\
\mathbf{F}_{\mathrm{b}}
\end{array}\right\}
$$

where $\Phi$ is the (N x n) matrix of retained or measured modes and $G_{r b}$ is a partition of the $(\mathrm{N} x \mathrm{~N})$ residual flexibility matrix defined in Eq. (2). If the displacements are partitioned into interior and boundary or interface degrees of freedom, Eq. (3) becomes

$$
\left\{\begin{array}{l}
\mathbf{u}_{\mathrm{i}} \\
\mathbf{u}_{\mathrm{b}}
\end{array}\right\}=\left[\begin{array}{cc}
\boldsymbol{\Phi}_{\mathrm{i}} & \mathbf{G}_{\mathrm{rib}_{\mathrm{b}}} \\
\boldsymbol{\Phi}_{\mathrm{b}} & \mathbf{G}_{\mathrm{rbb}}
\end{array}\right]\left\{\begin{array}{c}
\mathbf{q} \\
\mathbf{F}_{\mathrm{b}}
\end{array}\right\}
$$

By solving the lower partition of Eq. (4) for the boundary forces, and substituting the resulting expression back into Eq. (4), the interior physical displacements are obtained in terms of generalized interior and physical boundary displacements,

$$
u_{i}=\left(\Phi_{i}-G_{r_{i b}} G_{r b b}^{-1} \Phi_{b}\right) q+G_{r_{i b}} G_{r b b}^{-1} u_{b}
$$

Combining Eq. (5) with the identity $u_{b}=u_{b}$ yields the desired transformation for substructure displacements, 


$$
\left\{\begin{array}{l}
\mathbf{u}_{\mathrm{i}} \\
\mathbf{u}_{\mathrm{b}}
\end{array}\right\}=\left[\begin{array}{cc}
\Phi_{\mathrm{i}}-\mathbf{G}_{\mathrm{rib}_{\mathrm{ib}}} \mathbf{G}_{\mathrm{rbb}_{\mathrm{b}}}^{-1} \Phi_{\mathrm{b}} & \mathbf{G}_{\mathrm{rib}_{\mathrm{ib}}} \mathbf{G}_{\mathrm{rbb}_{\mathrm{bb}}}^{-1} \\
\mathbf{0} & \mathbf{I}
\end{array}\right]\left\{\begin{array}{c}
\mathbf{q} \\
\mathbf{u}_{\mathrm{b}}
\end{array}\right\}=\mathbf{T}\left\{\begin{array}{c}
\mathbf{q} \\
\mathbf{u}_{\mathrm{b}}
\end{array}\right\}
$$

where $T$ is an (Nx m) matrix and $m=n+n_{b}$, the number of retained modes plus the number of boundary dof.

The partitioned form of the undamped equation of motion for a substructure is

$$
\left[\begin{array}{cc}
\mathbf{M}_{\mathrm{ii}} & \mathbf{M}_{\mathrm{ib}} \\
\mathbf{M}_{\mathrm{bi}} & \mathbf{M}_{\mathrm{bb}}
\end{array}\right]\left\{\begin{array}{c}
\ddot{\mathbf{u}}_{\mathrm{i}} \\
\ddot{\mathbf{u}}_{\mathrm{b}}
\end{array}\right\}+\left[\begin{array}{cc}
\mathbf{K}_{\mathrm{ii}} & \mathbf{K}_{\mathrm{ib}} \\
\mathbf{K}_{\mathrm{bi}} & \mathbf{K}_{\mathrm{bb}}
\end{array}\right]\left\{\begin{array}{c}
\mathbf{u}_{\mathrm{i}} \\
\mathbf{u}_{\mathrm{b}}
\end{array}\right\}=\left\{\begin{array}{c}
\mathbf{0} \\
\mathbf{F}_{\mathrm{b}}
\end{array}\right\}
$$

and the corresponding partitioned form of the residual flexibility matrix is

$$
G_{r}=\left[\begin{array}{ll}
G_{r_{i i}} & G_{r_{i b}} \\
G_{r_{b i}} & G_{r_{b b}}
\end{array}\right]
$$

where $\mathbf{G}_{\mathrm{r}}$ is to be obtained using frequency response measurements of the free-free test article for the connect coordinates and shaker drive points (Ref. 2), or computed using Eqs. (1) and (2). The retained natural frequencies and mode shapes, $\omega_{n}^{2}$ and $\Phi_{n}$, are to be obtained from a free-boundary modal test, and correspond to subsets of the eigenvalues and eigenvectors of Eq. (8) with $F=0$. Applying the transformation defined in Eq. (6) to Eq. (8), the substructure reduced equation of motion becomes

$$
\overline{\mathbf{M}}\left\{\begin{array}{c}
\ddot{\mathbf{q}} \\
\ddot{\mathbf{u}}_{\mathrm{b}}
\end{array}\right\}+\overline{\mathbf{K}}\left\{\begin{array}{c}
\mathbf{q} \\
\mathbf{u}_{\mathrm{b}}
\end{array}\right\}=\mathbf{T}^{\mathbf{T}}\left\{\begin{array}{c}
\mathbf{0} \\
\mathbf{F}_{\mathbf{b}}
\end{array}\right\}
$$

where $\overline{\mathbf{M}}=\mathbf{T}^{\mathrm{T}} \mathbf{M T}$ and $\overline{\mathbf{K}}=\mathbf{T}^{\mathrm{T}} \mathbf{K T}$. Martinez, et. al (Ref. 5) showed that

$$
\begin{aligned}
& \overline{\mathbf{M}}=\left[\begin{array}{cc}
\mathbf{I}_{\mathrm{nn}}+\Phi_{\mathrm{nb}}^{\mathrm{T}} \mathbf{J}_{\mathrm{bb}} \Phi_{\mathrm{nb}} & -\Phi_{\mathrm{nb}}^{\mathrm{T}} \mathbf{J}_{\mathrm{bb}} \\
\text { sym. } & \mathbf{J}_{\mathrm{bb}}
\end{array}\right] \\
& \overline{\mathbf{K}}=\left[\begin{array}{cc}
\Omega_{\mathrm{nn}}^{2}+\Phi_{\mathrm{nb}}^{\mathrm{T}} \mathbf{G}_{\mathrm{rbb}}^{-1} \Phi_{\mathrm{nb}} & -\Phi_{\mathrm{nb}}^{\mathrm{T}} \mathbf{G}_{\mathrm{rbb}}^{-1} \\
\text { sym. } & \mathbf{G}_{\mathrm{rbb}}^{-1}
\end{array}\right]
\end{aligned}
$$

where $\Omega_{n n}$ is the diagonal matrix of retained or measured frequencies $\omega_{n}$, and $\Phi_{n b}$ is the boundary partition of the retained modes. Also in Eq. (10), $\mathbf{J}_{\mathrm{bb}}=\mathbf{G}_{\mathrm{rbb}}^{-1} \mathbf{H}_{\mathrm{bb}} \mathbf{G}_{\mathrm{rbb}}^{-1}$ and $H_{b b}=G_{r_{b}}^{T} M G_{r_{b}}$, where $G_{r_{b}}=\left[G_{r_{i b}} G_{r_{b b}}\right]^{T}$. Residual mass effects are contained in the boundary partition $\mathbf{H}_{\mathrm{bb}}$, and the full residual mass matrix is given by 


$$
\mathbf{H}_{\mathbf{r}}=\mathbf{G}_{\mathbf{r}}^{\mathrm{T}} \mathbf{M G}_{\mathbf{r}}
$$

In order to verify a constrained model, the corresponding constrained modes must be derived. This is accomplished using the present formulation by striking the rows and columns of the matrices in Eq. (10) for boundary dof, yielding

$$
\overline{\mathbf{M}}_{\mathrm{nn}} \ddot{\mathbf{q}}+\overline{\mathbf{K}}_{\mathrm{nn}} \mathbf{q}=\mathbf{0}
$$

where $\bar{M}_{\mathrm{nn}}=\left[\mathrm{I}_{\mathrm{nn}}+\Phi_{\mathrm{nb}}^{\mathrm{T}} \mathrm{J}_{\mathrm{bb}} \Phi_{\mathrm{nb}}\right]$ and $\overline{\mathrm{K}}_{\mathrm{nn}}=\left[\Omega_{\mathrm{nn}}^{2}+\Phi_{\mathrm{nb}}^{\mathrm{T}} \mathrm{G}_{\mathrm{rbb}}^{-1} \Phi_{\mathrm{nb}}\right]$, and both matrices are $(\mathrm{n} \times \mathrm{n})$. The eigenvalues $\omega_{c}^{2}$ calculated from Eq. (12) are the constrained frequencies, and the constrained modes are obtained by assembling the eigenvectors from Eq. (12), $\Phi_{\mathrm{nn}}$, into an ( $\mathrm{m} \times \mathrm{n})$ matrix and premultiplying by $\mathrm{T}$ from Eq. (6):

$$
\Phi_{\mathrm{c}}=\mathrm{T}\left[\begin{array}{c}
\Phi_{\mathrm{nn}} \\
\mathbf{0}
\end{array}\right]
$$

Since $T$ is $(\mathrm{N} \mathrm{x} \mathrm{m})$ and the partitioned mode shape matrix is ( $\mathrm{mxn}$ ), an $(\mathrm{N} \times \mathrm{n})$ matrix of constrained modes is obtained. The frequencies and mode shapes for the constrained structure, $\omega_{c}$ and $\Phi_{c}$, are used to obtain a verified constrained mathematical model.

\section{Frequency Response Function Approach for Measurement and Prediction of Residuals}

To provide an efficient means of comparing test residual measurements with analysis, the frequency response function (FRF) approach as presented by Rubin (Ref. 4) was utilized. In this method which is applicable to both analytical and test data, the displacement is written as a function of frequency,

$$
\mathbf{U}(\omega)=\mathbf{Y}(\omega) \mathbf{F}(\omega)
$$

where $\mathbf{Y}$ is the FRF matrix and $\mathbf{F}$ is the applied force as function of frequency. The residual FRF matrix, or residual function matrix as it will be denoted in this paper, is obtained by subtracting from the full FRF in Eq. (14) the modal FRF containing the rigid body modes and elastic free-free modes that are to be retained. The undamped modal FRF matrix is given by

$$
\mathbf{Y}_{m}(\omega)=-\Phi_{R} 1 / \omega^{2} M_{R}^{-1} \Phi_{R}^{T}+\Phi_{n} \Lambda_{n}^{-1} M_{n}^{-1} \Phi_{n}^{T}
$$

where $M_{n}$ is the generalized mass associated with the retained modes $\Phi_{n}$, and $\Lambda_{n}$ is the diagonal matrix $\omega_{n}^{2}-\omega^{2}$. The residual function matrix becomes

$$
\mathbf{Y}_{\mathrm{r}}(\omega)=\mathbf{Y}(\omega)-\mathbf{Y}_{\mathrm{m}}(\omega)
$$


which can be approximated over the frequency range of interest by the undamped form

$$
\mathbf{Y}_{\mathbf{r}}(\omega)=\mathbf{G}_{\mathbf{r}}+\omega^{2} \mathbf{H}_{\mathrm{r}}
$$

corresponding to Eqs. (2) and (11). For practical computations, residual functions are obtained individually rather than in matrix form. It is important to note that the FRF and residual functions described here are in displacement/force format. Residual flexibility for a particular residual function is the value of the function at zero frequency, as can be seen from Eq. (17). Each $G_{x}$ determined analytically in this way is equal to the corresponding value from Eq. (8).

\section{Results for Simple Structures}

Residual flexibility tests and analysis have been carried out for a simple beam and for the same beam with a flexible appendage. The appendage was designed to simulate a shuttle payload interface or trunnion. The simple structures were chosen to allow development of required procedures on a system whose dynamic characteristics are known with a high degree of confidence. In this manner, the analytical model, after adjustment to agree with measured free-free modes and frequencies, is taken as the "right answer" and provides the basis for assessing accuracy of residual measurements. To aid in determining the acceptable error in residual measurements, error analysis was performed for the beam with trunnion simulator.

\section{Simple Uniform Beam}

As shown in Fig. 1, the aluminum beam studied is 12' long with a 1" $\times 2$ " crosssection. The test article was suspended with flexible bungee cords, and 25 accelerometers were placed at 6" increments along the beam. A finite element model of the beam was developed having degrees-of-freedom corresponding to the measurement locations. In order to demonstrate the quality of the model and the modal measurements, a comparison of test and analysis mode shapes is shown in Fig. 2, and a frequency comparison is given in Table 1. Accuracy of experimental and analytical FRF can be seen in Fig. 3. The drive point FRF in Fig. 3 was obtained at the left end of the beam (Fig. 1), and the cross FRF was obtained by driving at the left end and obtaining the response at the point $1 / 4$ of the beam length from the left end.

In Fig. 4, comparisons of test and analytical residual functions for both the drive point and the quarter point are shown. For both test and analysis, the residuals are obtained by synthesizing a FRF from a subset of the mode shapes and subtracting it from the full FRF. Experimentally, the mode subset is simply the measured modes, while analytically a consecutive number of free-free modes corresponding to the number of measured modes is used.

It can be seen in Fig. 4 that the measured residual functions agree fairly well with analysis at higher frequencies, but that poor correlation is obtained at low frequencies. The spikes in the test data at system natural frequencies are not a cause of great concern because they are the result of fairly small differences in curve-fitted and measured FRF near the peak responses. Also, it must be emphasized that the data in Fig. 4 is presented in semilog format. It was found that by manipulating the damping values for each mode that the spikes could be reduced considerably, but at the expense of worse agreement at points away from the peaks, as shown in Fig. 5.

The poor agreement between test and analysis at low frequencies is a cause of concern. Since the model is believed to be correct (based on results shown in Figs. 2-3 and Table 1), the measurements are suspect. The quality of curve fitting in the modal analysis software should be the same at low frequencies as at higher frequencies (at least above about $5 \mathrm{~Hz}$ ). Thus, the discrepancies may possibly be due to the fact that at higher frequencies the 
difference in the full and synthesized FRF becomes greater due to neglected modes, and thus modal curve fitting errors become less important. As described later in the paper, a procedure was developed for working around the problem of poor or noisy data, both at low frequencies and at system natural frequencies.

\section{Simple Beam with Trunnion Simulator Attachment}

In an effort to obtain higher quality residual measurements, a screw simulating a shuttle payload trunnion was attached to the left end of the beam (Fig. 6). The rationale for this modification was that a trunnion structure having drastically different stiffness characteristics than the body of the beam would create a stiffness line as observed in drive point FRF of shuttle payloads. A clearly-defined stiffness line provides a prominent residual function that should be discernible even in the presence of curve fitting errors.

Mode shapes for the "trunnion" beam are similar to those for the uniform beam, with the differences being at the left end where the trunnion is attached. Figure 7 shows the fifth and sixth modes and demonstrates the quality of agreement between the test and model. In Table 2 a frequency comparison is shown with the mode shape correlation for the first 6 modes, and Fig. 8 shows comparison of test and analysis FRF. The first FRF in Fig. 8 is for excitation and response at the trunnion, the second for excitation at the trunnion and response at a point $6^{\prime \prime}$ from the left end, and the third for the same excitation point but response at midspan of the beam. In Fig. 9 the drive point FRF is shown in acceleration/force format. It can be seen that the function follows a stiffness line up to the first bending frequency of the trunnion.

As shown in Fig. 10 for the drive point residuals, good agreement is obtained between test and analysis. This clearly demonstrates the effect of the trunnion simulator, and the FRF stiffness line, on the behavior of the residual function. Again, the spikes are not a great cause of concern, though the disagreement at low frequencies still presents a problem. The cross residual functions in Fig. 10 show relatively poor agreement between test and analysis, though the trends match at higher frequencies. It is clear that for structures where off-diagonal terms of the residual flexibility matrix (Eq. (8)) are required, only the data at high frequencies ( $50 \mathrm{~Hz}$ or even higher for this structure) can be trusted.

An important conclusion to draw from Fig. 10 (and also Fig. 4) is that due to poor residual test data at low frequencies and at system natural frequencies, curve fitting is required to obtain accurate experimental values of residual flexibility. This is particularly obvious for cross or off-diagonal residual functions. In order to address the need for curve fitting of poor or noisy data, a general procedure for estimating residual flexibility values was developed as described in the following section.

\section{Statistical Least-Squares Curve Fitting Procedure for Identification of Experimental Residual Flexibility}

An investigation of curve fitting residual functions to determine residual flexibility values was conducted for the beam with trunnion simulator described in the last section. Since residual flexibility is defined as the value of a displacement/force (U/F) residual function at zero frequency, it is necessary to examine the functions in the U/F domain, as shown in Fig. 11, rather than the acceleration/force (A/F) domain shown in previous figures. As described previously, poor data is evident at low frequencies and system resonances. A second order polynomial curve fit of this data is required (excluding the first power coefficient) to determine the residual flexibility (constant coefficient) and the residual mass (second order coefficient), as shown in Eq. (17). Direct least-squares curve fitting of the data results in extreme divergence of the fit, as shown in Fig. 12. It is obvious that weighting must be applied to the data to emphasize regions of accurate measurements. 
A theoretical residual function in $\mathrm{U} / \mathrm{F}$ format is relatively flat at low frequencies and has slight upward curvature at higher frequencies. Variations of consecutive values of the residual function should therefore be small. When examining the residual function produced from the test data, the overall characteristics described previously can be seen. In the sections of poor data, consecutive residual values have large variations in magnitude. It is apparent, then, that a weighting function is needed that applies low weight to data points having large variation with respect to neighboring points, and high weight to data in regions of small variations.

Statistically, this can be expressed in terms of sample variance, where each sample consists of two or more neighboring data points.

By stepping through the test data, the variance of each data point with respect to the neighboring points can be calculated. The weighting value for each data point is set equal to the inverse of the variance assigned to that data point. This gives the desired effect that when the variances of neighboring data points are high the weighting function value is low and vise versa. Incorporating the weighting function into the least-squares curve fit, an acceptable fit is obtained as seen in Fig. 13. The residual function determined in this manner has the smooth characteristics previously described for theoretical U/F functions.

For the drive point residual function of the beam with trunnion simulator, different weighting matrices generated by examining two, three and four neighboring data points were used in the curve fit process. The error range for the residual flexibility term produced by examining different groups of data points was found to be from 0.4 to 2.5 percent. The examination of different groups of data points was performed because of the possibility of two or more consecutive data points having small variance, but the relative magnitude being considerably different from the characteristic curve. In addition, the frequency range of curve fitted data was varied to determine the effect on accuracy of the residual flexibility value. An example of the ranging of the curve fit is given in Fig. 14, and the error calculations for different sample sizes and frequency ranges are displayed in Table 3.

For the two residual functions generated from the responses taken at six inches from the left end of the beam and the middle point of the beam, Fig. 10, the errors compared to the theoretical value were high, about 25 percent. A closer examination of these two residual functions reveals that the functions are extremely ragged and in one case the characteristic curve of the test residual function does not fall on the theoretical curve. However, the curve fitting process provided the best fit of the residual test data. The high errors are due to limitations in the evaluation of the test frequency response function which produces the ragged data regions.

\section{Summary and Discussion}

Measurement and prediction of residual flexibility values for two simple structures, a uniform beam and beam with flexible appendage, have been demonstrated. The appendage was included to simulate a shuttle orbiter payload interface or trunnion. It was shown that frequency response functions (FRF) with dominant stiffness lines are needed in order to obtain accurate estimates of experimental residual flexibility. Trunnion drive point FRF for shuttle payloads exhibit this characteristic, pointing out the high potential of this technique for payload testing.

It was shown that test residual functions have regions of poor or noisy data, at low frequencies and at system resonances. The poor data appears to result from current limitations in modal parameter estimation software. A weighted least-squares curve fit that utilizes the inverse of data sample variance was applied to experimental residual functions to estimate residual flexibility. Results showed trunnion simulator drive point residual values within 0.4 to 2.5 percent of analytical predictions. The errors obtained from the curve fit of cross or off-diagonal residual functions were near 25 percent. Cross residual 
functions are typically not as critical as drive point functions and in some cases can be eliminated from the analysis.

References

1. Coleman, A. D., "A Mass Additive Technique for Modal Testing as Applied to the Space Shuttle ASTRO-1 Payload", Proceedings of the 6th International Modal Analysis Conference, February 1988, pp. 154-159.

2. Admire, J. R., Ivey, E. W., and Tinker, M. L., "Mass-Additive Modal Test Method for Verification of Constrained Structural Models", Proceedings of the 10th International Modal Analysis Conference, February 1992, accepted for publication.

3. MacNeal, R. H., "A Hybrid Method of Component Mode Synthesis", Computers and Structures, Vol. 1, 1971, pp. 581-601.

4. Rubin, S., "Improved Component Mode Representation for Structural Dynamic Analysis", AIAA Joumal, Vol. 13, 1975, pp. 995-1006.

5. Martinez, D. R., Carne, T. G. and Miller, A. K., "Combined Experimental/Analytical Modeling Using Component Mode Synthesis", Proceedings of the 25th Structures, Structural Dynamics and Materials Conference, May 1984, pp. 140-152.

6. Flanigan, C. C., "Test-Analysis Correlation of the Transfer Orbit Stage Modal Survey", Report 40864-8, Oct. 1988, SDRC, Inc., San Diego, CA.

7. Klosterman, A. L., and Lemon, J. R., "Dynamic Design Analysis via the Building Block Approach", Shock and Vibration Bulletin. No. 42, Pt. 1, Jan. 1972, pp. 97-104.

8. Smith, K., and Peng, C. Y., "SIR-C Antenna Mechanical System Modal Test and Model Correlation Report", JPL D-10694, Vol. 1, Jet Propulsion Laboratory, Pasadena, CA, April, 1993.

9. Blair, M. A., "Interface Characterization Modal Test Techniques," EM ATTIC 003, NASA Contract NAS8-38251 Final Report, Lockheed Missiles and Space Company, Sunnyvale, CA, Nov. 1991.

Nomenclature

A Transformation matrix for flexibility matrix

F External forces

G Flexibility

$\mathbf{G}_{\mathbf{r}} \quad$ Residual flexibility

H Mass associated with residual flexibility

K Substructure stiffness

$\overline{\mathbf{K}} \quad$ Reduced substructure stiffness 
m Number of retained modes plus boundary coordinates

M Substructure mass

$\overline{\mathbf{M}} \quad$ Reduced substructure mass

n Number of retained or measured modes

$n_{b} \quad$ Number of boundary coordinates

$\mathrm{N}$ Number of coordinates in unreduced substructure

q Generalized displacement

T Transformation to reduce substructure coordinates

u Physical substructure displacements

Y Frequency response function

$\mathbf{Y}_{\mathbf{r}} \quad$ Residual function

$\phi, \Phi$ Mode shapes

$\Lambda$ Diagonal frequency matrix for FRF formulation of residual technique

$\omega, \Omega$ Natural frequencies

subscripts

b Boundary coordinates

c Constrained substructure

cn Retained modes associated with connect points

f Flexible substructure

i Interior coordinates

m Modal

n Retained modes

r Residual

R Rigid body 


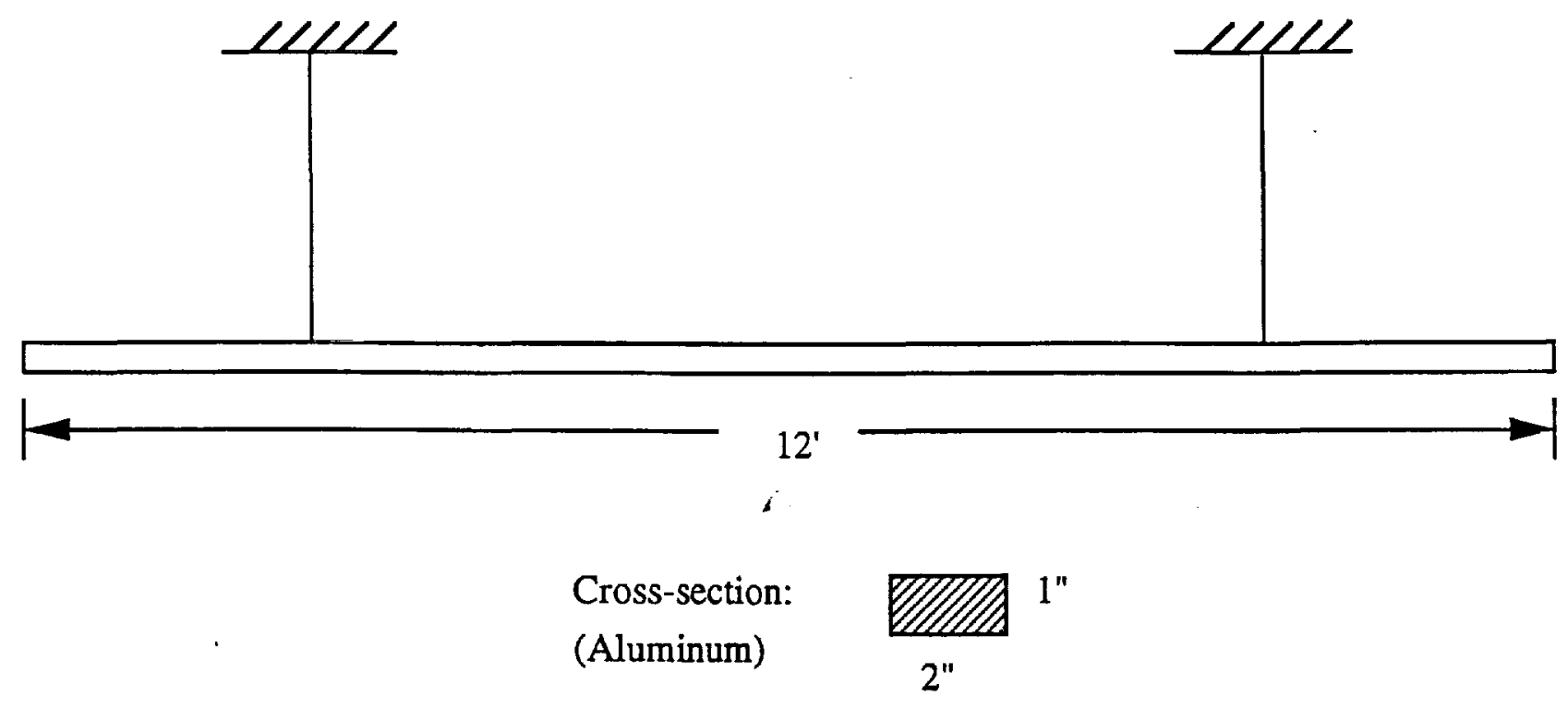

Figure 1. Beam Test Article

Table 1. Comparison of Test/Analysis Frequencies for Uniform Beam

\begin{tabular}{lllrl} 
& TEST & & \multicolumn{1}{c}{ ANAL } & CORR. \\
& & & & \\
1 & 10.0456 & 1 & 9.9338 & 0.99950 \\
2 & 27.6652 & 2 & 27.3716 & 0.99972 \\
3 & 54.2048 & 3 & 53.6386 & 0.99946 \\
4 & 89.5362 & 4 & 88.6362 & 0.99942
\end{tabular}



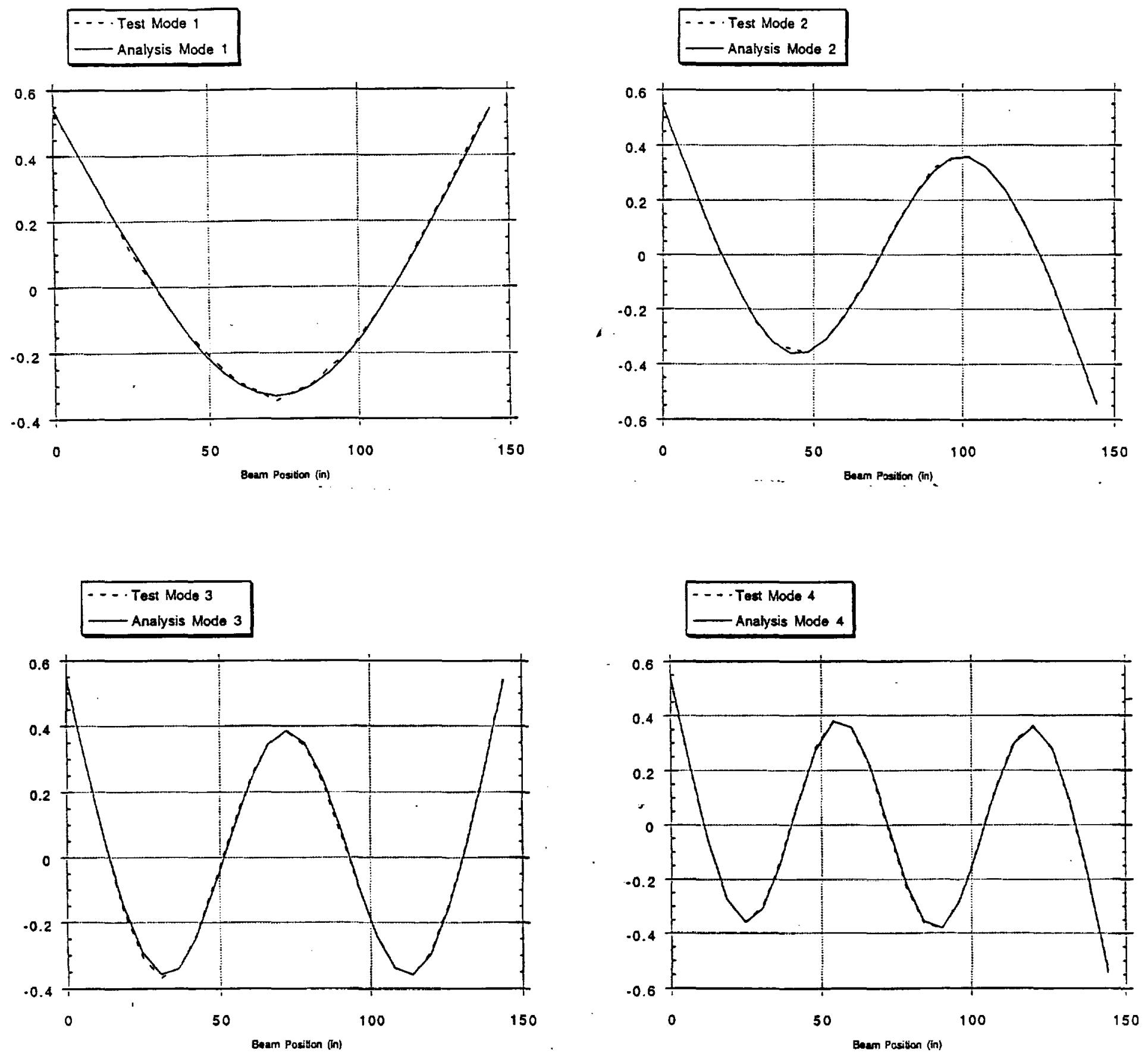

Figure 2. Comparison of Test/Analysis Mode Shapes
for Uniform Beam 

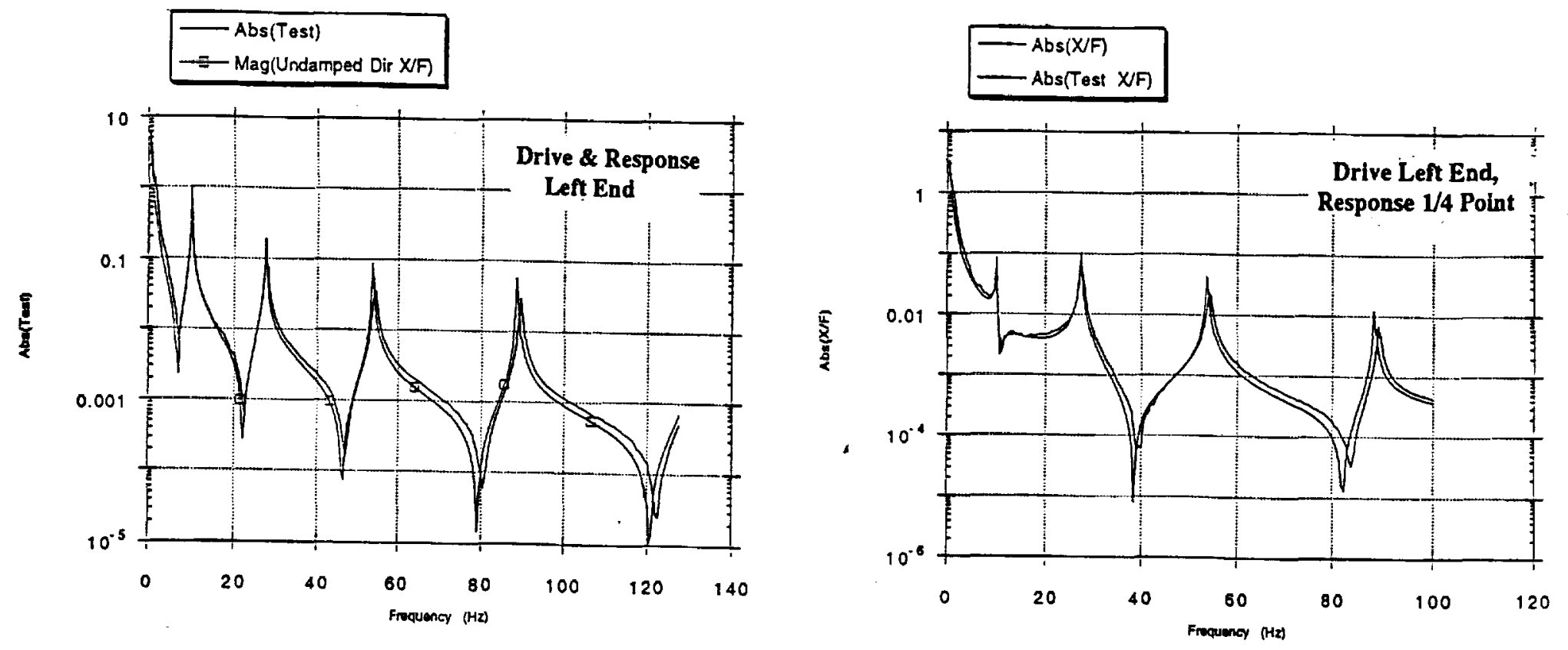

Figure 3. Comparison of Test/Analysis FRF for Uniform Beam
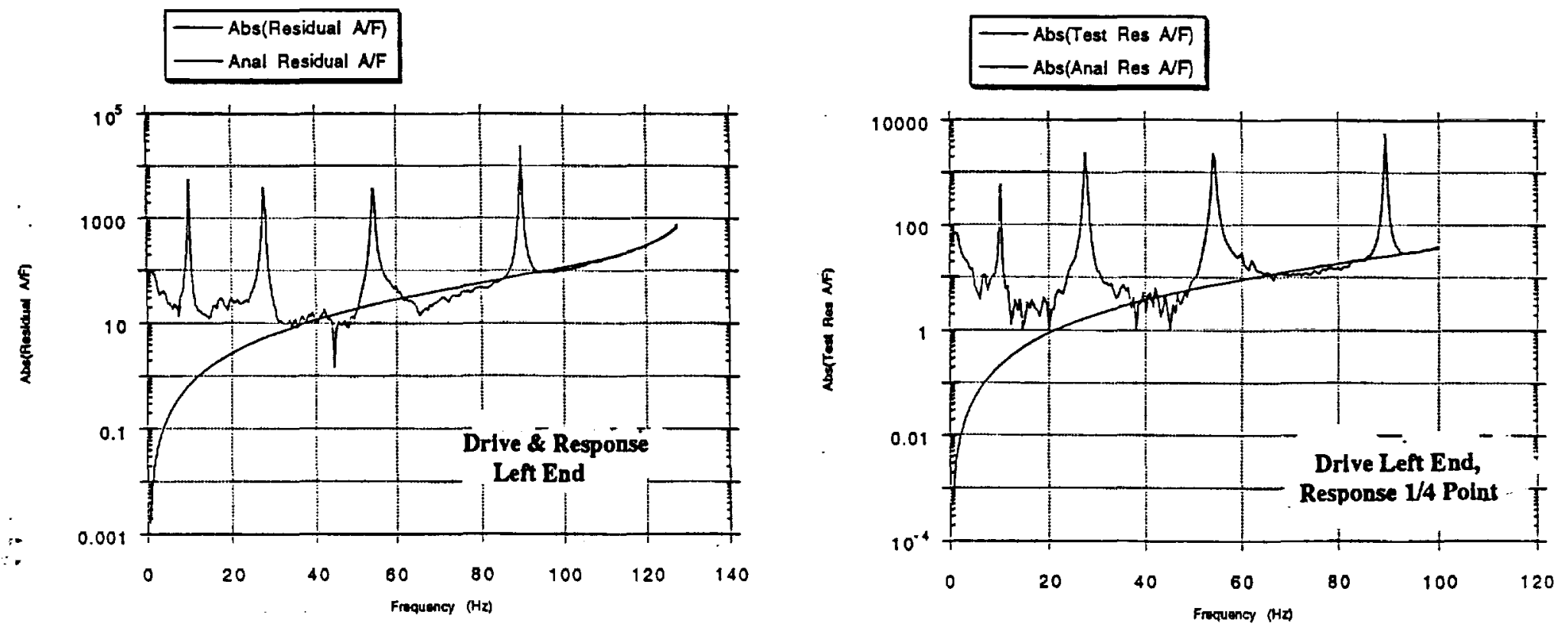

Figure 4. Test/Analysis Residual Functions for Uniform Beam 

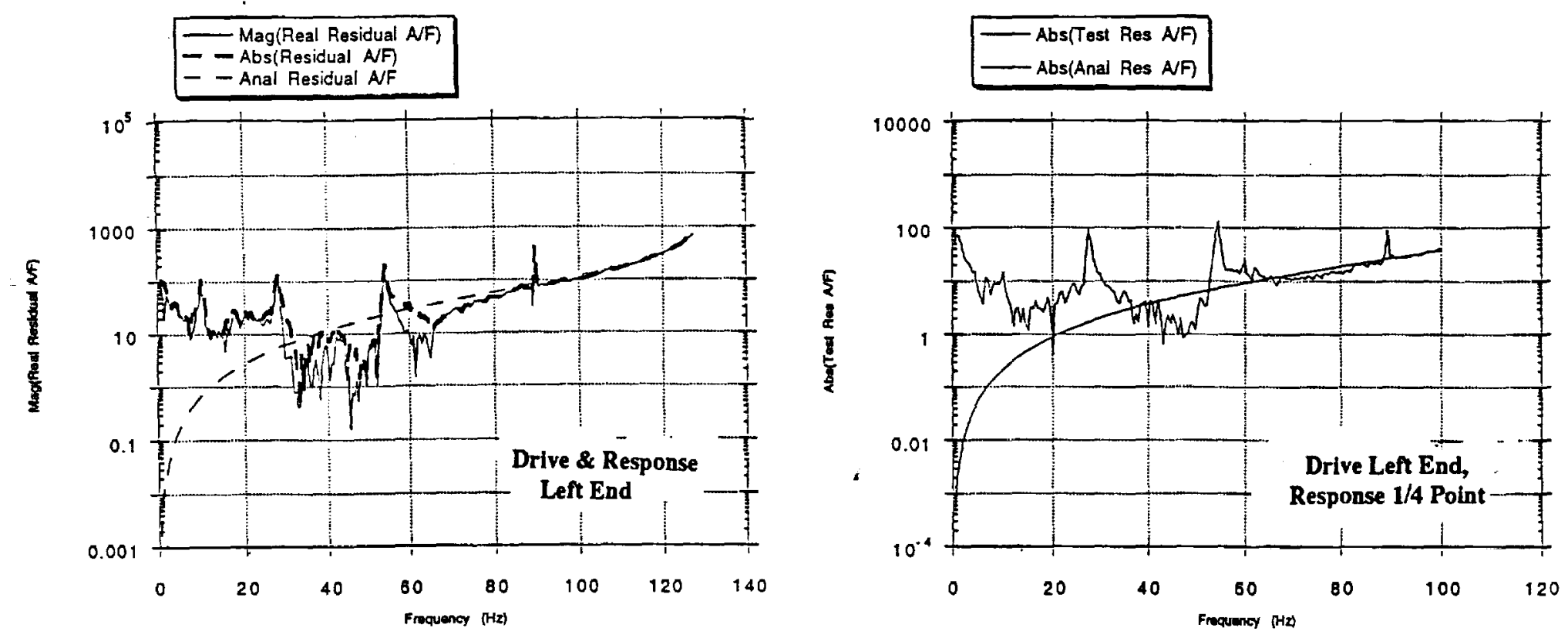

Figure 5. Test/Analysis Residual Functions for Modified Modal Damping

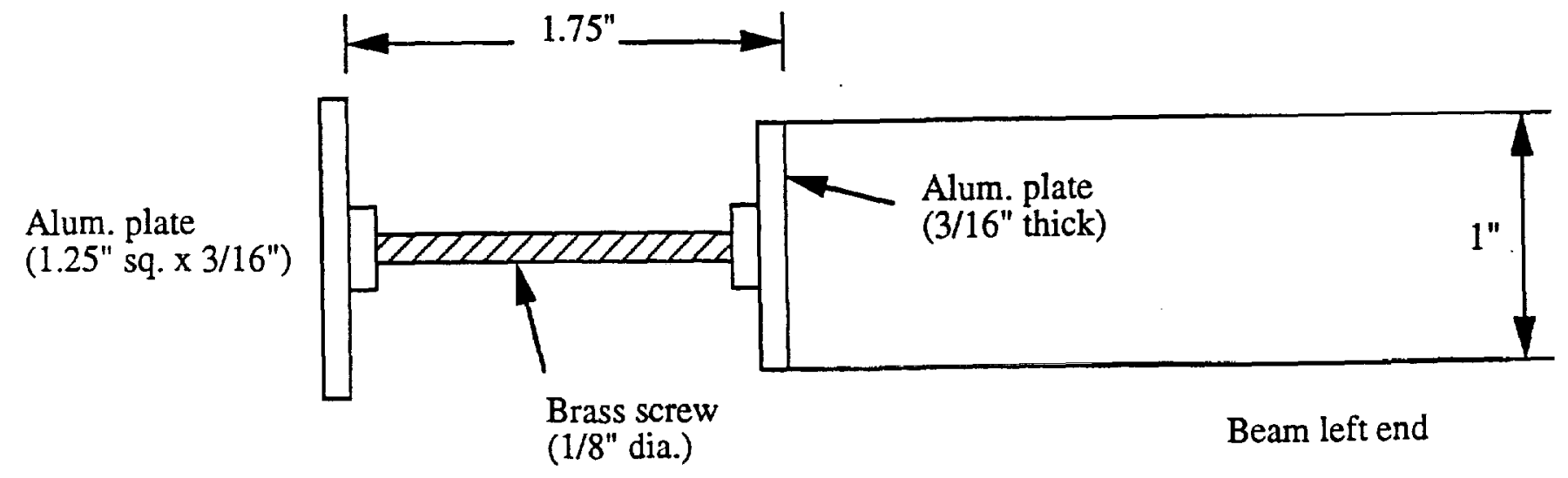

Figure 6. Modified Beam with Trunnion Attachment 

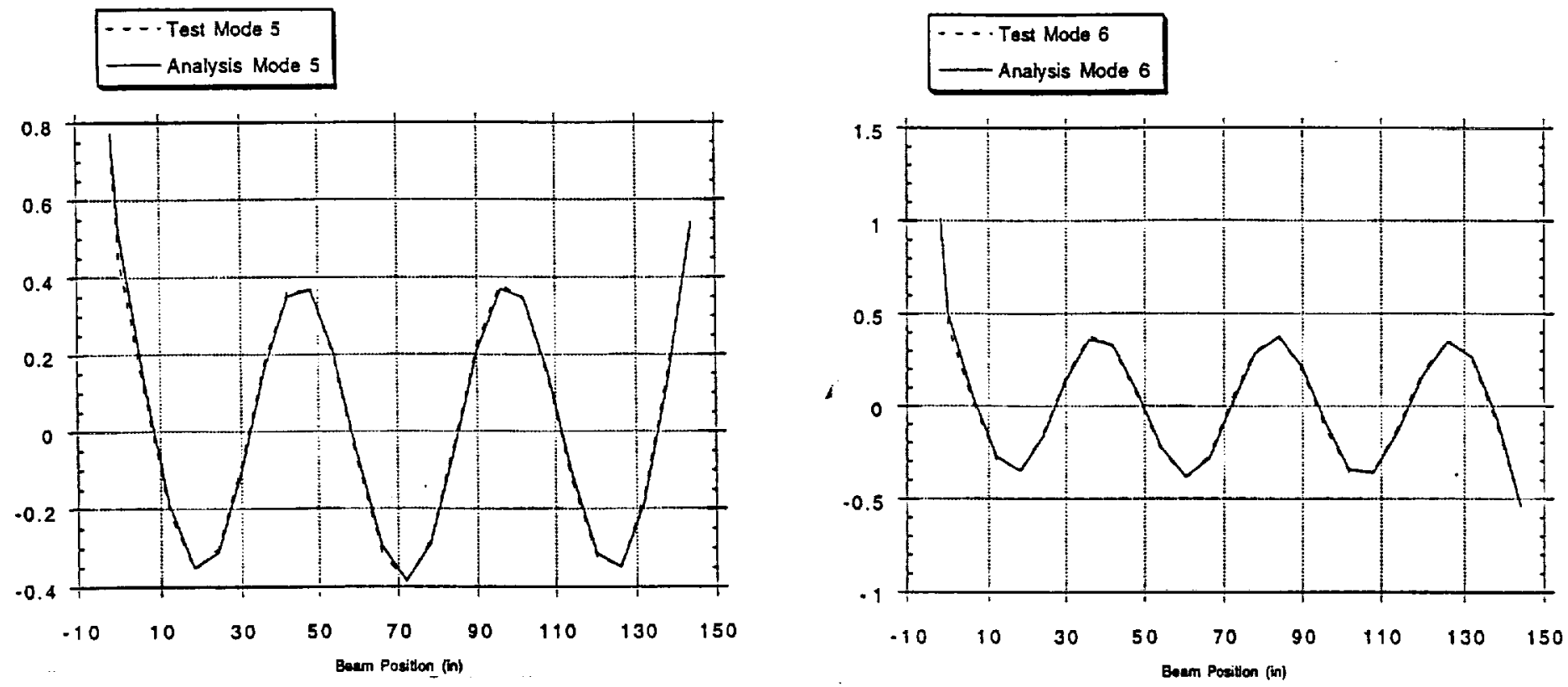

Figure 7. Comparison of Two Test/Analysis Modes for Beam with Trunnion Attachment

Table 2. Test/Analysis Frequencies for "Trunnion" Beam

TEST

$\begin{array}{rr}1 & 9.9888 \\ 2 & 27.4919 \\ 3 & 53.8265 \\ 4 & 88.9338 \\ 5 & 132.5650 \\ 6 & 184.7860\end{array}$
ANAL CORR.

$1 \quad 9.8874$

27.2392

53.3680

88.1604

131.5806

183.4944
0.99864

0.99940

0.99916

0.99872

0.99785

0.99698 

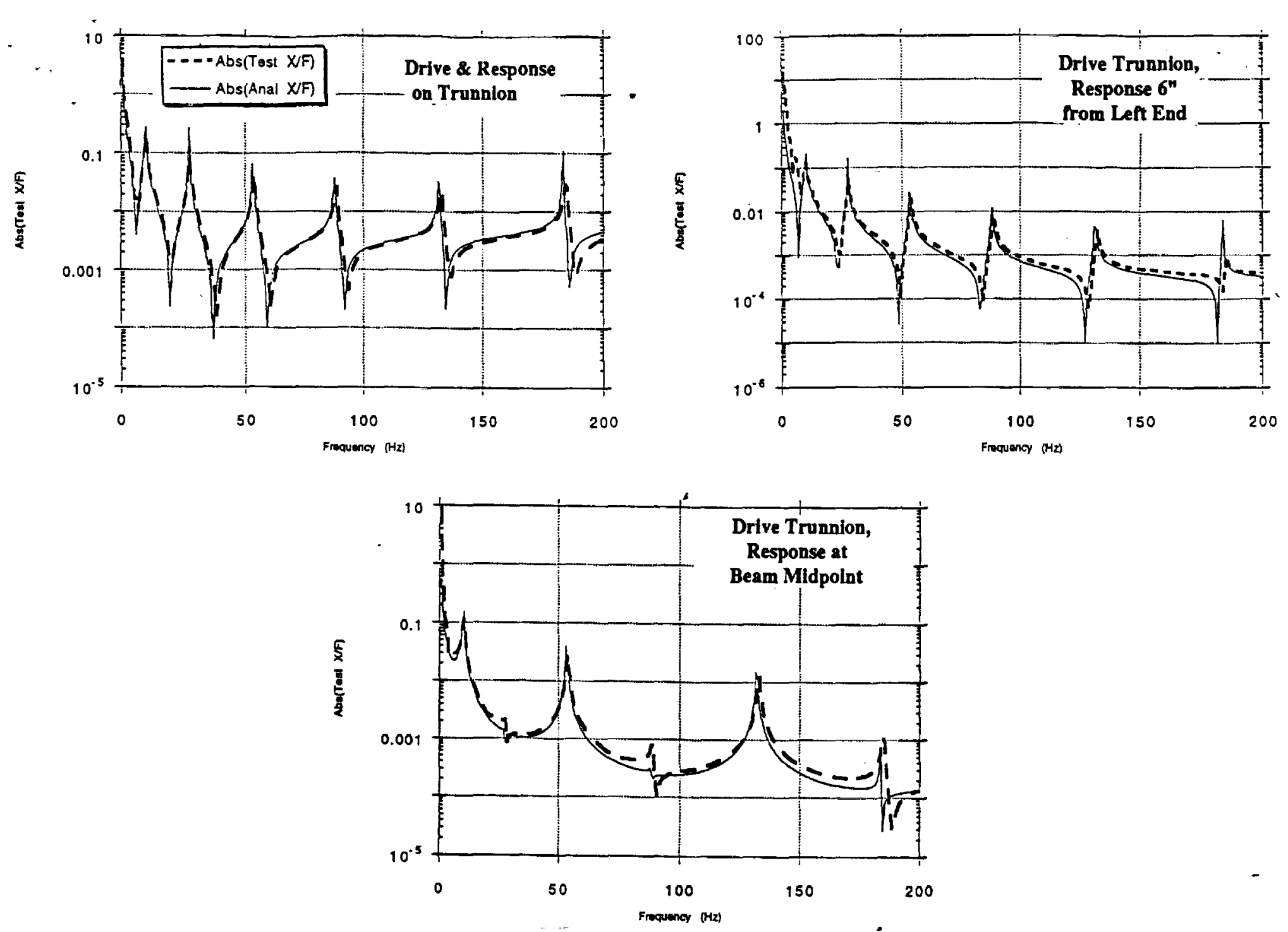

Figure 8. Comparison of Test/Analysis FRF for Beam with Trunnion Attachment

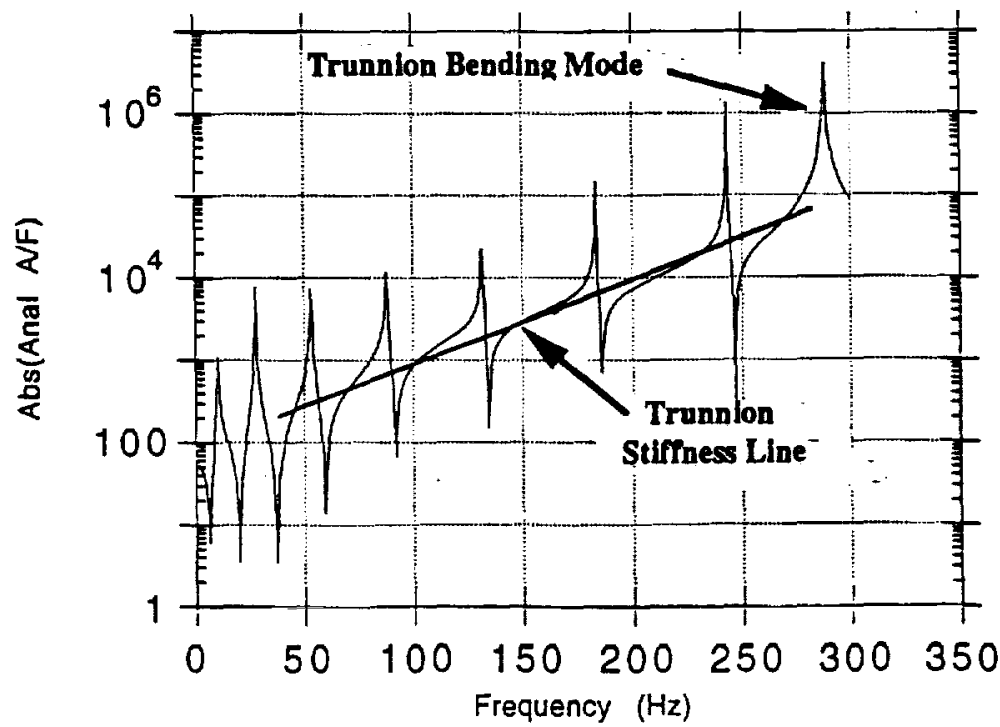

Figure 9. Drive Point FRF in A/F Format for Beam with Trunnion Attachment 

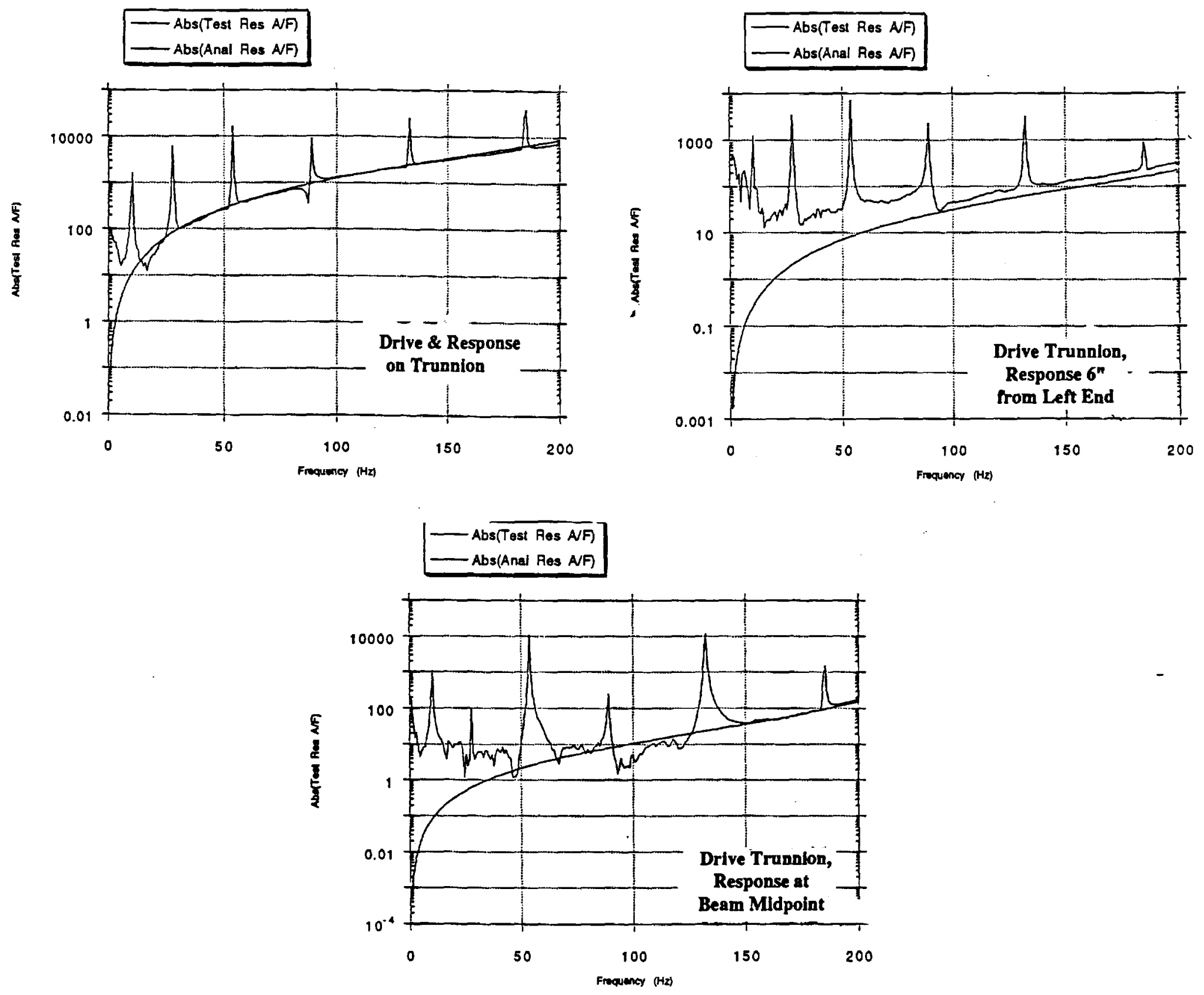

Figure 10. Test/Analysis Residual Functions for Beam with Trunnion Attachment 


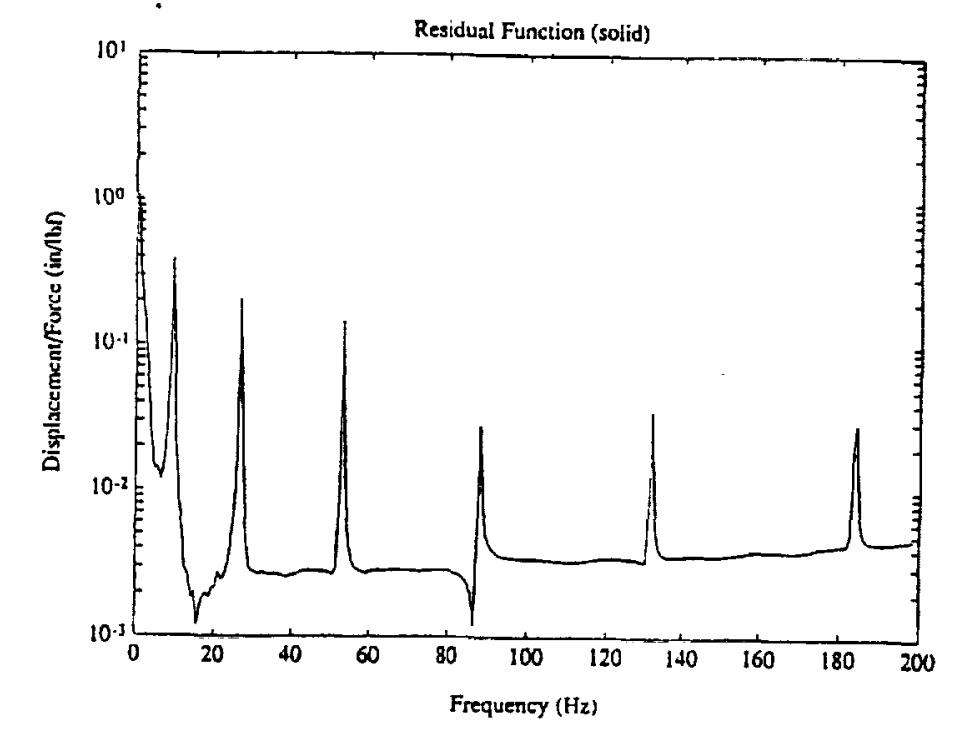

Eigure 11. Drive Point Residual Eunction

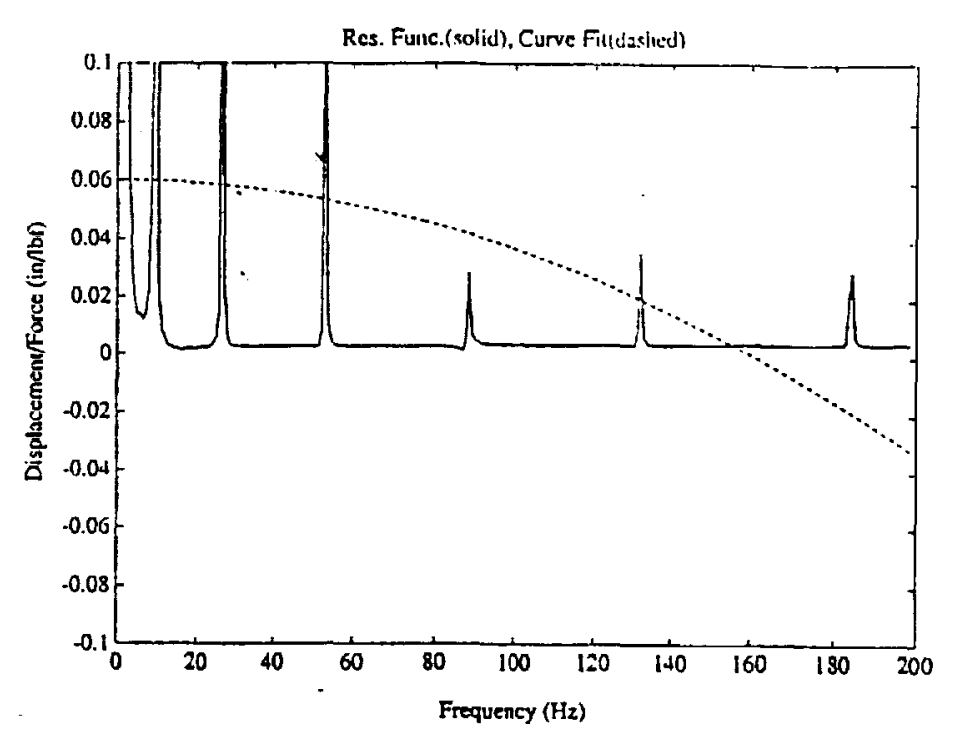

Figure 12. Direct Curve Fit
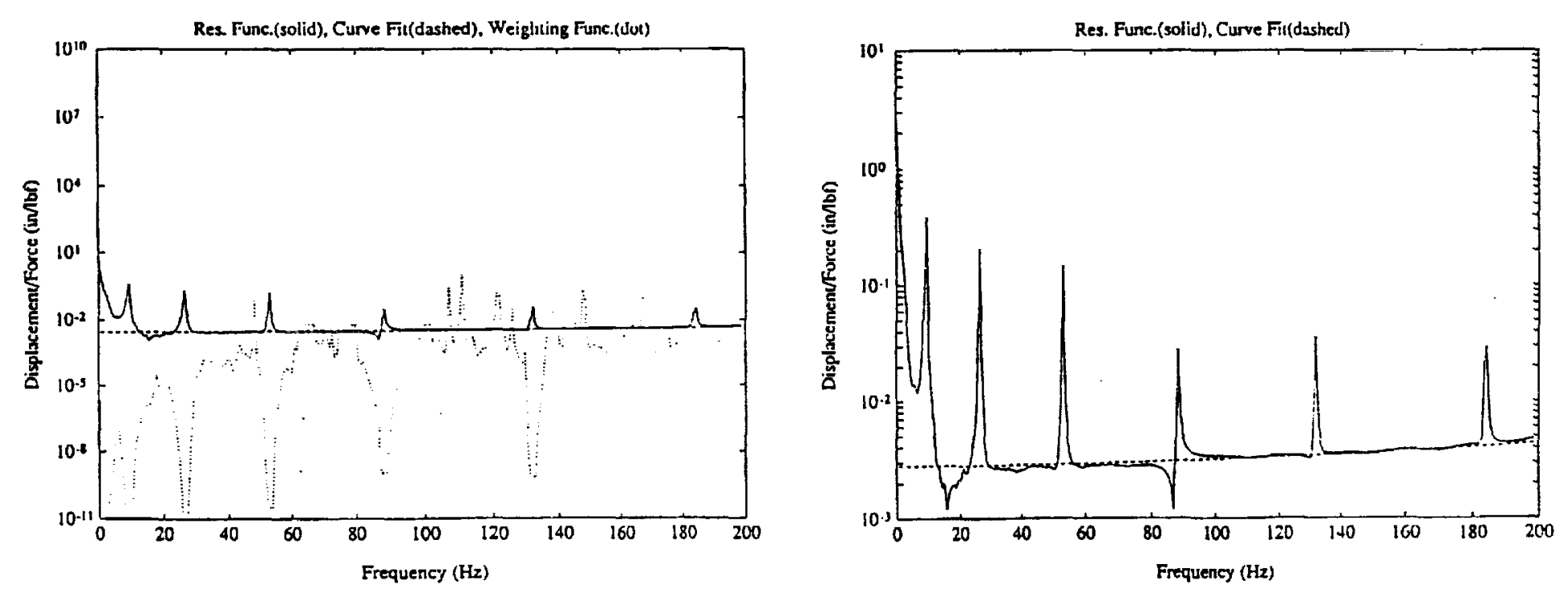

Figure 13. Weighted Curve Fit, With and without Weighting Function Plotted 


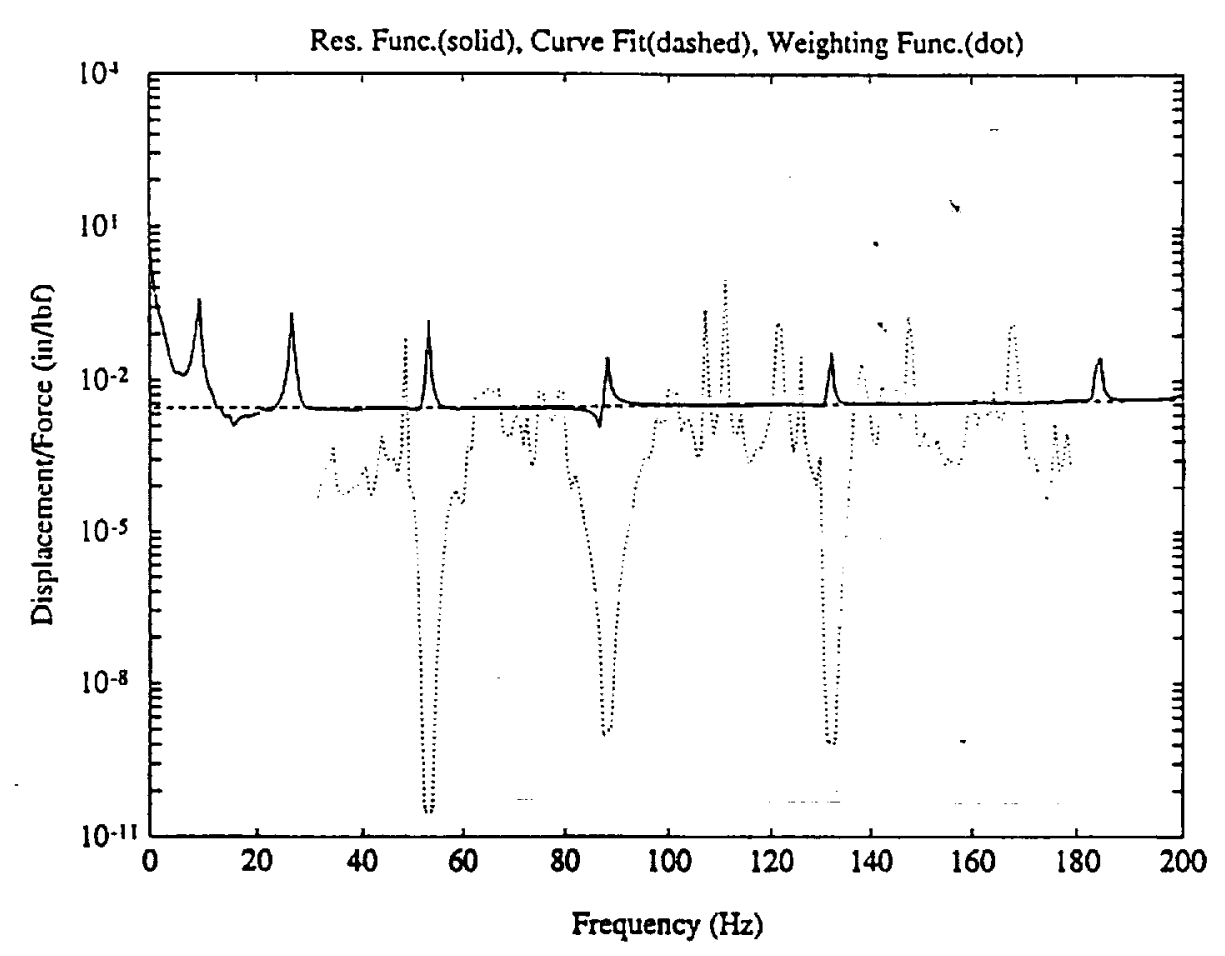

Figure 14. Ranging of Curve Fit

\begin{tabular}{cc|cc|ccc|cc}
$\begin{array}{c}\text { Lower } \\
\text { Freq }(\mathrm{Hz})\end{array}$ & $\begin{array}{c}\text { Upper } \\
\text { Freq }(\mathrm{Hz})\end{array}$ & 2 Points & \multicolumn{2}{c}{$\%$ Error } & 3 Points & \% Error & 4 Points & $\%$ Error \\
\hline Exact & & 0.0028309 & 0.00 & 0.0028309 & 0.00 & 0.0028309 & 0.00 \\
0 & 200 & 0.0027593 & 2.53 & 0.0028081 & 0.81 & 0.0027727 & 2.06 \\
30 & 200 & 0.0027594 & 2.53 & 0.0028082 & 0.80 & 0.0027731 & 2.04 \\
60 & 200 & 0.0027619 & 2.44 & 0.0028414 & 0.37 & 0.0027783 & 1.86 \\
& & & & & & & \\
0 & 180 & 0.0027596 & 2.52 & 0.0028136 & 0.61 & 0.0027824 & 1.71 \\
30 & 180 & 0.0027596 & 2.52 & 0.0028138 & 0.60 & 0.0027828 & 1.70 \\
60 & 180 & 0.0027621 & 2.43 & 0.0028489 & 0.64 & 0.0027885 & 1.50 \\
\hline
\end{tabular}

Table 3. Curve Fitting Errors of Residual Flexibility 